\title{
Exothermic dark matter for XENON1T excess
}

\author{
Hyun Min Lee \\ Department of Physics, Chung-Ang University, \\ Seoul 06974, Korea \\ CERN, Theory department, \\ 1211 Geneva 23, Switzerland \\ E-mail: hminlee@cau.ac.kr
}

AbSTRACT: Motivated by the recent excess in the electron recoil from XENON1T experiment, we consider the possibility of exothermic dark matter, which is composed of two states with mass splitting. The heavier state down-scatters off the electron into the lighter state, making an appropriate recoil energy required for the Xenon excess even for the standard Maxwellian velocity distribution of dark matter. Accordingly, we determine the mass difference between two component states of dark matter to the peak electron recoil energy at about $2.5 \mathrm{keV}$ up to the detector resolution, accounting for the recoil events over $E_{R}=2-3 \mathrm{keV}$, which are most significant. We include the effects of the phase-space enhancement and the atomic excitation factor to calculate the required scattering cross section for the Xenon excess. We discuss the implications of dark matter interactions in the effective theory for exothermic dark matter and a massive $Z^{\prime}$ mediator and provide microscopic models realizing the required dark matter and electron couplings to $Z^{\prime}$.

Keywords: Beyond Standard Model, Cosmology of Theories beyond the SM, Effective Field Theories, Gauge Symmetry

ARXiv EPrint: 2006.13183 


\section{Contents}

1 Introduction 1

2 Exothermic dark matter and electron recoil 2

2.1 Kinematics for exothermic dark matter 2

2.1.1 Heavy dark matter 4

2.1.2 Light dark matter 5

2.2 The event rate for electron recoil 6

3 The effective theory for exothermic dark matter 8

3.1 The effective interactions and the Xenon excess 8

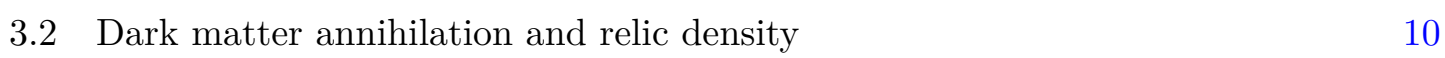

4 Microscopic models $\quad 13$

$\begin{array}{lll}4.1 & Z^{\prime} \text {-portal } & 15\end{array}$

4.2 Vector-like lepton portal 16

$\begin{array}{lll}5 & \text { Conclusions } & 17\end{array}$

\section{Introduction}

The nature of dark matter has been a long standing mystery for astrophysics and particle physics. Weakly Interacting Massive Particles (WIMPs) have been searched for in various direct detection, cosmic-ray as well as collider experiments, and the indirect probe or constraint from the early Universe and the intensity frontier has expanded the WIMP paradigm beyond weak scale.

Quite recently, a tantalizing hint has been announced for the potential dark matter signals from the electron recoil events in the recoil energy, $E_{R}=1-10 \mathrm{keV}$, from XENON1T experiment [1]. The origin of the Xenon excess has attracted interest from several groups [2-17]. A simple explanation with the solar axion or the neutrino magnetic dipole moment has been put forward from the XENON1T collaboration, but both cases are inconsistent with the star cooling constraints, because the electron coupling to the axion or the neutrino magnetic dipole moment required for the Xenon excess exceeds them by the order of magnitude [1].

In this article, we consider the possibility that exothermic dark matter transits from the heavy state to the light state in the event of the scattering with the electron. This possibility was already discussed in a different context in the literature with a motivation to explain the annual modulation signal at DAMA/LIBRA [18-20]. In this scenario, the down-scattering of dark matter makes the recoiled energy of the electron much larger than 
the one inferred from the elastic scattering between the non-relativistic dark matter in the standard halo model and the electron. We discuss the details of the kinematics of exothermic dark matter and calculate the scattering cross section for the Xenon events by including the phase-space enhancement for inelastic scattering and the atomic excitation factor for a small momentum transfer between dark matter and electron. We infer the required scattering cross section for dark matter as a function of dark matter mass at a fixed recoil energy near $E_{R}=2-3 \mathrm{keV}$ up to the detector resolution. We remark that there was a recent discussion on the monochromatic electron recoil spectrum in the case of $3 \rightarrow 2$ inelastic scattering between dark matter and electron [21].

We also provide the model-independent discussion with a massive $Z^{\prime}$ mediator on the effective model parameters explaining the Xenon excess and the dark matter relic density. We develop it for microscopic origins of the dark matter transition as well as the electron coupling, based on the $Z^{\prime}$-portal and the vector-like lepton portal.

A similar idea has been discussed in ref. [5] while this article is being finalized. Our results agree with theirs and complement with the detailed dynamics of exothermic dark matter such as the phase-space enhancement, the constraints from dark matter relic density, and concrete microscopic models realizing the scenario.

\section{Exothermic dark matter and electron recoil}

In this section, we begin with the kinematics for exothermic dark matter in the case of downscattering off the electron. Then, we calculate the event rate for the electron recoil in the Xenon atoms by including the phase-space enhancement and the atomic excitation factor.

\subsection{Kinematics for exothermic dark matter}

We first consider the inelastic scattering between dark matter and electron, $\chi_{1} e \rightarrow \chi_{2} e$, where two dark fermions, $\chi_{1}$ and $\chi_{2}$, have a small mass difference, $\Delta m=m_{\chi_{1}}-m_{\chi_{2}}>0$, which is the exothermal condition for the transition in the dark matter states. We assume that only $\chi_{1}$ or both $\chi_{1}$ and $\chi_{2}$ account for the observed relic density for dark matter. The up-scattering process, $\chi_{2} e \rightarrow \chi_{1} e$, is also possible if kinematically allowed in the tail of the dark matter velocity distribution, for instance, at $v \sim 0.1$ for $\Delta m \sim \mathrm{keV}$. But, we regard the up-scattering process as being effectively forbidden for the standard halo model. Thus, we focus on the down-scattering process, $\chi_{1} e \rightarrow \chi_{2} e$, in the following discussion.

The electrons bound to Xenon atoms need the ionization energy to be excited to free electrons, and they carry a nonzero velocity, $v_{e} \sim Z_{\text {eff }} \alpha \sim 10^{-2}$, where $Z_{\text {eff }}=1$ for outer shell electrons and it is larger for inner shells. Moreover, since electrons are bound to the atom, the entire atom recoils. In this case, the energy transferred to the electron can be obtained in terms of the energy lost in the dark matter and the nuclear recoil energy [22], as follows,

$$
\begin{aligned}
\Delta E_{e} & =-\Delta E_{\mathrm{DM}}-\Delta E_{N} \\
& =\Delta m\left(1-\frac{1}{2}\left(\frac{m_{\chi_{1}}}{m_{\chi_{2}}}\right) v^{2}\right)+\frac{m_{\chi_{1}}}{m_{\chi_{2}}} \vec{q} \cdot \vec{v}-\frac{\vec{q}^{2}}{2 \mu_{\chi_{2} N}}
\end{aligned}
$$




$$
\begin{aligned}
& =\Delta m\left(1-\frac{1}{2}\left(\frac{m_{\chi_{1}}}{m_{\chi_{2}}}\right) v^{2}\right)-\frac{1}{2 \mu_{\chi_{2} N}}\left(\vec{q}-\frac{m_{\chi_{1}}}{m_{\chi_{2}}} \mu_{\chi_{2} N} \vec{v}\right)^{2}+\frac{1}{2} \mu_{\chi_{2} N}\left(\frac{m_{\chi_{1}}}{m_{\chi_{2}}}\right)^{2} v^{2} \\
& \leq \Delta m\left(1-\frac{1}{2}\left(\frac{m_{\chi_{1}}}{m_{\chi_{2}}}\right) v^{2}\right)+\frac{1}{2} \mu_{\chi_{2} N}\left(\frac{m_{\chi_{1}}}{m_{\chi_{2}}}\right)^{2} v^{2}
\end{aligned}
$$

where $\mu_{\chi_{2} N}$ is the reduced mass for dark matter $\chi_{2}$ and the nucleus, $\mu_{\chi_{2} N}=$ $m_{\chi_{2}} m_{N} /\left(m_{\chi_{2}}+m_{N}\right)$. Thus, the electron recoil energy is maximized if $\vec{q}=\frac{m_{\chi_{1}}}{m_{\chi_{2}}} \mu_{\chi_{2} N} \vec{v}$. Due to the bound electrons, the recoil energy of the ionized electron is given by $E_{R}=\Delta E_{e}-E_{B i}$ where $E_{B i}$ is the binding energy of the electron. We note that there is a crucial difference from the elastic scattering, in that the upper bound on the electron energy is increased by the mass difference between dark matter components.

For $\Delta m=0$, the typical momentum transfer in the elastic scattering between dark matter and electron is given by $q_{\text {typ }} \sim \mu_{\chi e} v_{\text {rel }} \sim m_{e} v_{e} \sim Z_{\text {eff }}(4 \mathrm{keV})$ for $m_{\chi} \gtrsim 1 \mathrm{MeV}$. But, for $\Delta m \neq 0$, the approximate momentum transfer given by eq. (2.12) becomes almost independent of the velocity and $q^{2} \sim m_{e} \Delta m \gg q_{\mathrm{typ}}^{2} \sim m_{e}^{2} v_{e}^{2}$ for $\Delta m \gg m_{e} v_{e}^{2} \sim 0.05 \mathrm{keV}$. Then, we can also ignore the nuclear recoil energy in the above formula (2.1), because $\Delta E_{N}=\frac{\vec{q}^{2}}{2 \mu_{\chi_{2} N}} \sim \frac{m_{e}}{m_{\chi_{2}}}(\Delta m) \ll \Delta m$ for $m_{e} \ll m_{\chi_{2}}<m_{N}$.

In the following discussion, we include a nonzero velocity of electron in the lab frame and ignore the nuclear recoil effects to show the salient effect of the mass splitting for the electron recoil energy. We take the electron and dark matter $\chi_{1}$ to have nonzero velocities in the lab frame, $v_{e}$ and $v$, respectively, then their initial kinetic energies are given by $E_{e}=\frac{1}{2} m_{e} v_{e}^{2}$ and $E_{0}=\frac{1}{2} m_{\chi_{1}} v^{2}$. From the energy conservation, the total energy after scattering satisfies the following relation,

$$
m_{\chi_{2}}+\frac{p^{2}}{2 M_{2}}+\frac{p_{\mathrm{cm}}^{2}}{2 \mu_{2}}=m_{\chi_{1}}+E_{0}+E_{e}
$$

where $\mu_{2}$ is the reduced mass of the dark matter-electron system after scattering, given by $\mu_{2}=m_{e} m_{\chi_{2}} /\left(m_{e}+m_{\chi_{2}}\right)$, and $M_{2}=m_{e}+m_{\chi_{2}}, p$ is the total 3-momentum and $p_{\mathrm{cm}}$ is the dark matter momentum in the center of mass frame after scattering. Then, we can solve eq. (2.2) to get

$$
p_{\mathrm{cm}}=\sqrt{2 \mu_{2}\left(\Delta m+E_{0}+E_{e}-\frac{p^{2}}{2 M_{2}}\right)}
$$

with $\Delta m=m_{\chi_{1}}-m_{\chi_{2}}$. On the other hand, we also get the total 3-momentum in terms of the initial kinetic energies,

$$
p^{2}=2 m_{\chi_{1}} E_{0}+2 m_{e} E_{e}+4 \cos \alpha \sqrt{m_{e} m_{\chi_{1}} E_{0} E_{e}}
$$

with $\alpha$ being the angle between electron and dark matter velocities.

The electron recoil energy is given by the difference between the electron kinetic energies before and after scattering,

$$
\begin{aligned}
\Delta E_{e} & =\frac{1}{2 m_{e}}\left(\frac{m_{e}}{M_{2}} \vec{p}-\vec{p}_{\mathrm{cm}}\right)^{2}-E_{e} \\
& =\frac{1}{2 m_{e}}\left(\frac{m_{e}^{2}}{M_{2}^{2}} p^{2}+p_{\mathrm{cm}}^{2}-\frac{2 m_{e}}{M_{2}} p p_{\mathrm{cm}} \cos \theta\right)-E_{e}
\end{aligned}
$$


where $\theta$ is the scattering angle in the center of mass frame. Then, using eq. (2.3), we obtain the exact expression for the electron recoil energy in terms of as

$$
\begin{aligned}
\Delta E_{e}= & \frac{m_{e}}{2 M_{2}^{2}} p^{2}+\frac{\mu_{2}}{m_{e}}\left(\Delta m+E_{0}+E_{e}\right)-\frac{\mu_{2}}{2 m_{e} M_{2}} p^{2}-E_{e} \\
& -\frac{p}{M_{2}} \cos \theta \sqrt{2 \mu_{2}\left(\Delta m+E_{0}+E_{e}\right)-\frac{\mu_{2}}{M_{2}} p^{2}} .
\end{aligned}
$$

On the other hand, the 3-momentum transfer $q$ is also given by

$$
q^{2}=2 m_{e} \Delta E_{e}+m_{e}^{2} v_{e}^{2}-2 m_{e} v_{e} \cos \psi \sqrt{2 m_{e} \Delta E_{e}}
$$

where $\psi$ is the scattering angle of the electron in the lab frame. Here, we note that $q^{2}=2 m_{e} \Delta E_{e}$ only for the electron at rest, which is the standard relation between the recoil energy and the electron, when the target electron is at rest.

In the following subsections, we divide our discussion on the electron recoil energy, depending on the masses of dark matter components.

\subsubsection{Heavy dark matter}

First, we take the limit with $m_{\chi_{1}} E_{0} \gtrsim m_{e} E_{e}$, which is the case for $m_{\chi_{1}} \gtrsim m_{e}\left(v_{e} / v\right)^{2} \sim$ $10 \mathrm{MeV}$, i.e. heavy dark matter. In the next section, our detailed discussion on the Xenon electron events will be based on this case. Then, we can approximate the total 3-momentum to be $p^{2} \simeq 2 m_{\chi_{1}} E_{0}$ from eq. (2.4). As a result, from eq. (2.6), we obtain the approximate electron recoil energy as follows,

$$
\Delta E_{e} \simeq \frac{\mu_{2} \Delta m}{m_{e}}\left(1-\frac{E_{0}}{M_{2}}\right)-\frac{m_{e}}{M_{2}} E_{e}+\frac{\mu_{2}^{2} E_{0}}{m_{e} m_{\chi_{2}}}\left[\left(1+\frac{m_{\chi_{1}}}{m_{\chi_{2}}}\right)-2 \sqrt{\kappa} \cos \theta\right]
$$

with

$$
\begin{aligned}
\kappa & \equiv \frac{m_{\chi_{1}}}{m_{\chi_{2}}}\left(1+\frac{m_{\chi_{2}} E_{e}}{\mu_{2} E_{0}}\right)+\frac{m_{\chi_{1}} \Delta m}{\mu_{2} E_{0}}\left(1-\frac{E_{0}}{M_{2}}\right) \\
& =\frac{m_{\chi_{1}}}{m_{\chi_{2}}}\left(1+\frac{m_{\chi_{2}} m_{e} v_{e}^{2}}{m_{\chi_{1}} \mu_{2} v^{2}}\right)+\frac{2 \Delta m}{\mu_{2} v^{2}}\left(1-\frac{\mu_{2} v^{2}}{2 m_{e}} \frac{m_{\chi_{1}}}{m_{\chi_{2}}}\right) .
\end{aligned}
$$

In the case for heavy dark matter, taking the limits for $\Delta m \ll m_{e} \ll m_{\chi_{1}}$ and $\Delta m \gg m_{e} v_{e}^{2} \sim 50 \mathrm{eV}$, we can approximate eq. (2.9) as

$$
\begin{aligned}
\kappa & \simeq \frac{m_{e} v_{e}^{2}+2 \Delta m}{m_{e} v^{2}} \\
& \simeq \frac{\Delta m}{\frac{1}{2} m_{e} v^{2}} \simeq 2.2 \times 10^{4}\left(\frac{220 \mathrm{~km} / \mathrm{s}}{v}\right)^{2}\left(\frac{\Delta m}{3 \mathrm{keV}}\right) .
\end{aligned}
$$

Indeed, choosing $\Delta m \simeq 3 \mathrm{keV}$ from Xenon electron recoil energy and $v \simeq 220 \mathrm{~km}$ from the averaged velocity of dark matter in the standard halo model, we obtain $\kappa \gg 1$. In this case, from eq. (2.8), the electron recoil energy becomes simplified to

$$
\begin{aligned}
\Delta E_{e} & \simeq \Delta m\left(1-\frac{1}{2}\left(\frac{m_{\chi_{1}}}{m_{\chi_{2}}}\right) v^{2}\right)-\frac{m_{e}^{2} v_{e}^{2}}{2 m_{\chi_{1}}}+m_{e} v^{2}(1-\sqrt{\kappa} \cos \theta) \\
& \simeq \Delta m\left(1-\frac{2}{\sqrt{\kappa}} \cos \theta\right) .
\end{aligned}
$$


On the other hand, from eq. (2.7), for $\Delta E_{e} \simeq \Delta m \gg m_{e} v_{e}^{2}$, we can also get the approximate result for the momentum transfer as

$$
q^{2} \simeq 2 m_{e} \Delta E_{e} \simeq 2 m_{e} \Delta m\left(1-\frac{2}{\sqrt{\kappa}} \cos \theta\right)
$$

Therefore, either the recoil energy or the momentum transfer depend little on either the dark matter and electron velocities or the scattering angle. As a result, we get a tiny momentum transfer fixed by the mass difference, for which the atomic excitation factor becomes important [3, 5, 23-26], as will be discussed later.

For $\Delta m \ll m_{e} \ll m_{\chi_{1}}$ but with $\kappa \simeq 1$, we have the following approximate results,

$$
\begin{aligned}
\Delta E_{e} & \simeq \Delta m+m_{e} v^{2}(1-\cos \theta), \\
q^{2} & \simeq 2 m_{e}^{2} v^{2}(1-\cos \theta) .
\end{aligned}
$$

In this case, the recoil energy is bounded by $2 m_{e} v^{2} \simeq 5.5 \times 10^{-4} \mathrm{keV} \gg \Delta m$ for $v \sim$ $220 \mathrm{~km} / \mathrm{s}$, which is too small to account for the Xenon experiment. Thus, we focus on the regime with $\kappa \gg 1$ in the following discussion, which is sufficient for explaining the Xenon excess.

\subsubsection{Light dark matter}

In the opposite limit with $m_{\chi_{1}} E_{0} \lesssim m_{e} E_{e}$, that is, $m_{\chi_{1}} \lesssim m_{e}\left(v_{e} / v\right)^{2} \sim 10 \mathrm{MeV}$, that is, light dark matter, we can approximate the total momentum as $p^{2} \simeq 2 m_{e} E_{e}$. In this case, from eq. (2.6), we get the approximate recoil energy as follows,

$$
\Delta E_{e} \simeq \frac{\mu_{2}}{m_{e}}\left(\Delta m+E_{0}\right)-\frac{2 \mu_{2}}{M_{2}} E_{e}(1+\sqrt{\tilde{\kappa}} \cos \theta)
$$

with

$$
\begin{aligned}
\tilde{\kappa} & \equiv 1+\frac{m_{e}}{\mu_{2} E_{e}}\left(\Delta m+E_{0}\right) \\
& =1+\frac{1}{\mu_{2} v_{e}^{2}}\left(2 \Delta m+m_{\chi_{1}} v^{2}\right) .
\end{aligned}
$$

In the case for light dark matter, taking the limits for $\Delta m \ll m_{e} \ll m_{\chi_{1}}$ and $m_{\chi_{1}} v^{2} \lesssim$ $100 \mathrm{eV} \ll \Delta m$, we can approximate eq. (2.16) as

$$
\tilde{\kappa} \simeq \frac{\Delta m}{\frac{1}{2} m_{e} v_{e}^{2}} \simeq 10^{2}\left(\frac{10^{-2} c}{v_{e}}\right)^{2}\left(\frac{\Delta m}{3 \mathrm{keV}}\right) .
$$

Then, from eq. (2.15), the electron recoil energy becomes simplified to

$$
\begin{aligned}
\Delta E_{e} & \simeq \Delta m\left(1+\frac{m_{\chi_{1}} v^{2}}{2 \Delta m}\right)-\frac{m_{e}^{2} v_{e}^{2}}{m_{\chi_{1}}}(1+\sqrt{\tilde{\kappa}} \cos \theta) \\
& \simeq \Delta m\left(1-\frac{2 m_{e}}{m_{\chi_{1}}} \frac{1}{\sqrt{\tilde{\kappa}}} \cos \theta\right) .
\end{aligned}
$$


On the other hand, from eq. (2.7), for $\Delta E_{e} \simeq \Delta m \gg m_{e} v_{e}^{2}$, we can also get the approximate result for the momentum transfer as

$$
q^{2} \simeq 2 m_{e} \Delta E_{e} \simeq 2 m_{e} \Delta m\left(1-\frac{2 m_{e}}{m_{\chi_{1}}} \frac{1}{\sqrt{\tilde{\kappa}}} \cos \theta\right)
$$

Then, as in the case for heavy dark matter, the electron recoil energy depends little on either the dark matter and electron velocities or the scattering angle, but it is determined dominantly by the mass difference.

\subsection{The event rate for electron recoil}

We begin with the general expression for the event rate per target mass [27, 28], given by

$$
d R=\frac{\rho_{\chi_{1}} v}{m_{\chi_{1}} m_{T}} d \sigma f_{1}(v) d v
$$

where $m_{T}$ is the target nucleus mass and $f_{1}(v)=\frac{4 v^{2}}{v_{0}^{3} \sqrt{\pi}} e^{-v^{2} / v_{0}^{2}}$ with $v_{0}=220 \mathrm{~km} / \mathrm{s}$ for the Maxwellian velocity distribution of dark matter and $\int_{0}^{\infty} f_{1}(v) d v=1$, and $\rho_{\chi_{1}}$ is the local energy density of dark matter, which is given by $\rho_{\chi_{1}}=0.4 \mathrm{GeV} / \mathrm{cm}^{3}$ if $\chi_{1}$ occupies the full dark matter.

We note that the differential scattering cross section for the inelastic scattering is given by

$$
\frac{d \sigma}{d E_{R}}=\frac{2 m_{e} \bar{\sigma}_{e}}{q_{+}^{2}-q_{-}^{2}} \int_{q_{-}}^{q_{+}} a_{0}^{2} q^{\prime} d q^{\prime} K\left(E_{R}, q^{\prime}\right) P^{2}(v),
$$

where $\bar{\sigma}_{e}$ is the total cross section in the limit of elastic scattering for the fixed momentum transfer at $q=1 / a_{0}$, with $a_{0}=\frac{1}{\alpha m_{e}}$ being the Bohr radius, $K\left(E_{R}, q^{\prime}\right)$ is the atomic enhancement factor, and $P^{2}(v)$ is the phase space factor, which is unity for the elastic scattering. Then, from eqs. (2.20) and (2.21), we obtain the differential event rate per target mass as

$$
\frac{d R}{d E_{R}}=\frac{2 m_{e} \bar{\sigma}_{e} \rho_{\chi_{1}}}{m_{\chi_{1}} m_{T}} K_{\mathrm{int}}\left(E_{R}\right) \int_{v_{\min }}^{\infty} \frac{v P^{2}(v)}{q_{+}^{2}-q_{-}^{2}} f_{1}(v) d v
$$

where the integrated atomic enhancement factor is given by

$$
K_{\mathrm{int}}\left(E_{R}\right)=\int_{q_{-}}^{q_{+}} a_{0}^{2} q^{\prime} d q^{\prime} K\left(E_{R}, q^{\prime}\right),
$$

and $v_{\min }$ is the minimum velocity of dark matter required for a given recoil energy $E_{R}$. We note that the total recoil energy is also deposited significantly near $E_{R} \sim \mathrm{keV}$ to ionize the electrons bound to the Xenon atoms, and the atomic excitation factor can be important for a small momentum transfer [3, 5, 23-26]. As a result, we get the event rate per detector as

$$
R_{D}=M_{T} \int_{E_{T}}^{\infty} \frac{d R}{d E_{R}} d E_{R}
$$

where $E_{T}$ is the detector threshold energy and $M_{T}$ is the fiducial mass of the detector, given by $M_{T} \simeq 4.2 \times 10^{27}\left(M_{T} /\right.$ tonne $) m_{T}$ for Xenon. 
Now we apply the general result in eq. (2.22) for the case with down-scattering dark matter. Assuming that dark matter mass $m_{\chi_{1}}$ is greater than $10 \mathrm{MeV}$, we can use our results for heavy dark matter in section 2.1.1. Then, we take $\kappa \gg 1$, for which the recoil energy is appreciable. In this case, we obtain the phase space factor $P^{2}(v)$ in eq. $(2.22)$ as

$$
P^{2}(v) \simeq \sqrt{1+\frac{2 \Delta m}{\mu_{1} v^{2}}} \simeq \sqrt{\frac{2 \Delta m}{m_{e}}} \frac{1}{v} .
$$

Then, using eq. (2.22) with $q_{ \pm}^{2} \simeq 2 m_{e} \Delta m\left(1 \pm \frac{2}{\sqrt{\kappa}}\right)$ from eq. (2.12), we obtain the differential event rate for $E_{-}<E_{R}<E_{+}$with $E_{ \pm}=\Delta m\left(1 \pm \frac{2}{\sqrt{\kappa}}\right)$ as

$$
\frac{d R}{d E_{R}} \simeq \frac{\bar{\sigma}_{e} \rho_{\chi_{1}}}{2 m_{e} m_{\chi_{1}} m_{T}} K_{\mathrm{int}}\left(E_{R}\right) \int_{0}^{v_{\max }} \frac{f_{1}(v)}{v} d v \theta\left(E_{R}-E_{-}\right) \theta\left(E_{+}-E_{R}\right)
$$

where $v_{\min }=0, v_{\max }=\sqrt{\frac{2 \Delta m}{m_{e}}}$ at $\kappa=1$. For $E_{+}-E_{-} \ll E_{ \pm}$, we can approximate $\theta\left(E_{R}-E_{-}\right) \theta\left(E_{+}-E_{R}\right) \simeq\left(E_{+}-E_{-}\right) \delta\left(E_{R}-\Delta m\right)$. Therefore, we can rewrite eq. (2.22) as

$$
\frac{d R}{d E_{R}} \simeq\left(\frac{2 \Delta m}{m_{e}}\right)^{1 / 2} \frac{\bar{\sigma}_{e} \rho_{\chi_{1}}}{m_{\chi_{1}} m_{T}} K_{\mathrm{int}}\left(E_{R}\right) \delta\left(E_{R}-\Delta m\right) \int_{0}^{v_{\max }} f_{1}(v) d v
$$

We note that for $\Delta m \gg 1.3 \times 10^{-4} \mathrm{keV}$, we have $v_{\max } \gg v_{0}$, resulting in $\int_{0}^{v_{\max }} f_{1}(v) d v \simeq 1$. Therefore, we find that there is no Boltzmann suppression due to an enhancement factor $P^{2}(v)$ in eq. (2.25), as compared to the case with elastic scattering.

Consequently, from eq. (2.24) with eq. (2.27), we get the total event rate per Xenon detector with $m_{T}=m_{X e}$ as

$$
\begin{aligned}
R_{D} \simeq & \left(\frac{M_{T} \bar{\sigma}_{e} \rho_{\chi_{1}}}{m_{\chi_{1}} m_{T}}\right)\left(\frac{2 \Delta m}{m_{e}}\right)^{1 / 2} K_{\mathrm{int}}(\Delta m) \\
\simeq & 50\left(\frac{M_{T}}{\text { tonne }-\mathrm{yrs}}\right)\left(\frac{K_{\mathrm{int}}(\Delta m)}{2.6}\right)\left(\frac{\rho_{\chi_{1}}}{0.4 \mathrm{GeV} \mathrm{cm}^{-3}}\right) \\
& \times\left(\frac{\bar{\sigma}_{e} / m_{\chi_{1}}}{1.2 \times 10^{-43} \mathrm{~cm}^{2} / \mathrm{GeV}}\right)\left(\frac{\Delta m}{2.5 \mathrm{keV}}\right)^{1 / 2}
\end{aligned}
$$

where we has used the normalization for the integrated atomic excitation factor at $E_{R} \simeq$ $2 \mathrm{keV}$ and for the momentum transfer peaked at $q \simeq 50 \mathrm{keV}$.

For comparison to the experimental data, the mono-energetic event rate can be convoluted with the detector resolution by

$$
\frac{d R_{D}}{d E_{R}}=\frac{R_{D}}{\sqrt{2 \pi} \sigma} e^{-\left(E_{R}-\Delta m\right)^{2} /\left(2 \sigma^{2}\right)} \alpha(E)
$$

where $\sigma$ is the detector resolution, which varies between $20 \%$ at $E=2 \mathrm{keV}$ and $6 \%$ at $E=30 \mathrm{keV}$, and $\alpha(E)$ is the signal efficiency [1]. For $E_{R}=2-10 \mathrm{keV}$, the signal efficiency is given by $\alpha(E) \sim 0.7-0.9[1]$.

The XENON1T excess is most significant from the electrons at $E_{R}=2-3 \mathrm{keV}$ with the detector resolution being about $\sigma=0.4 \mathrm{keV}$, so we take the recoil energy of the monoenergetic electron in our model to be $E_{R} \simeq \Delta m \simeq 2.5 \mathrm{keV}$. Moreover, from $\alpha(E) \simeq 0.8$ at 


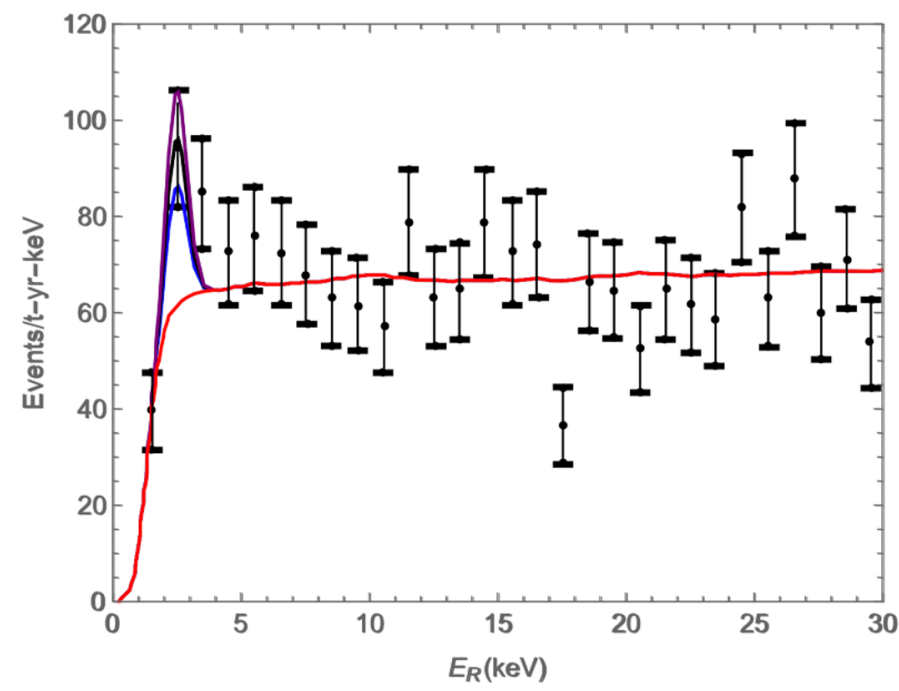

Figure 1. The event rate for electron recoil as a function of recoil energy $E_{R}$ in $\mathrm{keV}$. We have taken $\Delta m=2.5 \mathrm{keV}$ and $\bar{\sigma}_{e} / m_{\chi_{1}}=1.0,1.4,1.8 \times 10^{-43} \mathrm{~cm}^{2}$ from bottom to top lines (purple, black and blue. The red line is the background model used by Xenon experiment [1].

$E_{R} \simeq 2.5 \mathrm{keV}$, we need to rescale the total event rate per detector in eq. (2.28) by a factor 0.8. Therefore, taking into account the total exposure in XENON1T for SR1, which is 0.65 tonne-yrs [1], and for $\rho_{\chi_{1}}=0.4 \mathrm{GeV} \mathrm{cm}^{-3}$, we can get about 50 events near $E_{R}=2-3 \mathrm{keV}$ for the XENON1T electron recoil events for $\bar{\sigma}_{e} / m_{\chi_{1}} \simeq 3.4 \times 10^{-43} \mathrm{~cm}^{2} / \mathrm{GeV}$.

In figure 1 , we depict the expected event rate for the signal + background in units of events per $\mathrm{t}-\mathrm{yr}-\mathrm{keV}$ as a function of the electron recoil energy in $\mathrm{keV}$ in our model with exothermic dark matter. The expected event rates are shown in blue lines for $\Delta m=2.5 \mathrm{keV}$ and $\bar{\sigma}_{e} / m_{\chi_{1}}=1.0,1.4,1.8 \times 10^{-43} \mathrm{~cm}^{2}$, from bottom to top, and the red line corresponds to the background model used by Xenon experiment [1]. The values of the mass difference and $\bar{\sigma}_{e} / m_{\chi_{1}}$ can be varied for the global fit with the observed electron recoil spectrum, but the monochromatic shape of the signal from exothermic dark matter above the flat background remains the same up to the detector resolution. We can compare our case to the existing global fit of the XENON electron recoil spectrum for the case with axion-like dark matter whose mass is fixed to $m_{a}=2.3 \pm 0.2 \mathrm{keV}$ with $3.0 \sigma$ local significance [1].

\section{The effective theory for exothermic dark matter}

We continue to discuss the effective theory for exothermic dark matter in the presence of a massive $Z^{\prime}$ mediator and constrain the parameter space for the $Z^{\prime}$ couplings and the mass parameters from the Xenon excess. For completeness, we also provide the formulas for dark matter annihilation cross sections in the effective theory and comment on the compatibility of the Xenon excess with the correct relic density.

\subsection{The effective interactions and the Xenon excess}

We consider two Majorana dark matter fermions, $\chi_{1}$ and $\chi_{2}$, with different masses, $m_{\chi_{1}}>$ $m_{\chi_{2}}$, and a massive dark gauge boson $Z^{\prime}$ with mass $m_{Z^{\prime}}$. We take the effective Lagrangian 
with $Z^{\prime}$ couplings to dark fermions, electron and electron neutrino, in the following form,

$$
\begin{aligned}
\mathcal{L}_{\text {eff }}= & \left(g_{Z^{\prime}} Z_{\mu}^{\prime} \bar{\chi}_{2} \gamma^{\mu}\left(v_{\chi}+a_{\chi} \gamma^{5}\right) \chi_{1}+\text { h.c. }\right)+g_{Z^{\prime}} Z_{\mu}^{\prime} \bar{e}\left(v_{e}+a_{e} \gamma^{5}\right) e \\
& +g_{Z^{\prime}} Z_{\mu}^{\prime} \bar{\nu} \gamma^{\mu}\left(v_{\nu}+a_{\nu} \gamma^{5}\right) \nu
\end{aligned}
$$

where $v_{i}, a_{i}$ with $i=\chi, e, \nu$ are constant parameters. In the next subsection, we will show a microscopic model for the above effective interactions. For $\Delta m<m_{Z^{\prime}}$ and $\Delta m<2 m_{e}$, there is no tree-level decay process for the dark matter fermion $\chi_{1}$.

For $a_{e} \neq 0$, the effective vertex interaction for one $Z^{\prime}$ and two photons, $Z_{\mu}^{\prime}(q)-A_{\lambda}\left(q_{1}\right)-$ $A_{\sigma}\left(q_{2}\right)$, with $q=q_{1}+q_{2}$, is induced by the electron loops, taking the following form for $q^{2} \ll 4 m_{e}^{2}[35,36]$,

$$
\Gamma^{\mu \lambda \sigma}\left(q, q_{1}, q_{2}\right) \simeq \epsilon^{q_{1} \lambda \sigma \mu} \cdot \frac{a_{e} e^{2} g_{Z^{\prime}}}{4 \pi^{2}}\left(1+\frac{q^{2}}{12 m_{e}^{2}}\right) .
$$

Then, the heavy state of dark matter can decay into the lighter state of dark matter and two photons, with the decay rate given by

$$
\Gamma\left(\chi_{1} \rightarrow \chi_{2} \gamma \gamma\right) \simeq \frac{a_{e}^{2}\left(v_{\chi}^{2}+a_{\chi}^{2}\right) e^{4} g_{Z^{\prime}}^{2}}{2560 \pi^{7}} \frac{(\Delta m)^{5}}{m_{Z^{\prime}}^{4}} .
$$

Therefore, in this case, the lifetime of the dark fermion $\chi_{1}$ is much longer than the age of the Universe for perturbative effective couplings. However, the diffuse X-ray background [37] puts the bound on the lifetime of the dark fermion $\chi_{1}$ to $\tau_{\chi_{1}}>10^{24}$ sec, which gives rise to

$$
\left|a_{e}\right| g_{Z^{\prime}} \sqrt{v_{\chi}^{2}+a_{\chi}^{2}}<2.5 \times 10^{-6}\left(\frac{2.5 \mathrm{keV}}{\Delta m}\right)^{5 / 2}\left(\frac{m_{Z^{\prime}}}{1 \mathrm{GeV}}\right)^{2} .
$$

As a result, we need a small axial vector coupling to the electron to satisfy the X-ray bounds for exothermic dark matter and light $Z^{\prime}$ mediator. As we will discuss in the next section, some microscopic models with vector-like leptons can induce a suppressed axial vector coupling to the electron.

Moreover, there is another loop process for the three-photon decay channel, $\chi_{1} \rightarrow$ $\chi_{2}+3 \gamma$, but the corresponding decay rate is highly suppressed by $\Gamma \propto(\Delta m)^{13} /\left(m_{Z^{\prime}}^{4} m_{e}^{8}\right)[5]$, thus being consistent with X-ray bounds [37].

If the neutrino couplings to $Z^{\prime}$ are nonzero, the dark matter fermion $\chi_{1}$ would decay into a neutrino pair via the off-shell $Z^{\prime}$ gauge boson, which is bounded by the lifetime of dark matter for explaining the XENON1T electron recoil excess. The decay rate of the dark fermion $\chi_{1}$ is given [34] by

$$
\Gamma\left(\chi_{1} \rightarrow \chi_{2} \nu \bar{\nu}\right) \simeq \frac{N_{\nu} G_{F}^{\prime 2}(\Delta m)^{5}}{30 \pi^{3}}\left(v_{\chi}^{2}+3 a_{\chi}^{2}\right)\left(v_{\nu}^{2}+a_{\nu}^{2}\right)
$$

where $N_{\nu}$ is the number of neutrinos coupled to $Z^{\prime}$, and $G_{F}^{\prime} \equiv g_{Z^{\prime}}^{2} /\left(\sqrt{2} m_{Z^{\prime}}^{2}\right)$. Then, for $\Delta m=2.5 \mathrm{keV}$ and $v_{\chi}=-a_{\chi}=\frac{1}{2}$, the lifetime of the dark fermion $\chi_{1}$ is longer than the age of the Universe, as far as

$$
G_{F}^{\prime} \sqrt{N_{\nu}\left(v_{\nu}^{2}+a_{\nu}^{2}\right)}<2.4 \times 10^{-6} \mathrm{GeV}^{-2} .
$$


Nonetheless, neutrino experiments such as Super-Kamiokande [38] would constrain the lifetime of dark matter to $\tau_{\chi_{1}}>10^{24} \mathrm{sec}$ for $\Delta m>0.1 \mathrm{MeV}$ [39-42]. But, for $\Delta m=2.5 \mathrm{keV}$, the energy of the produced neutrinos is below those of solar neutrinos, so there is no current bound from neutrino experiments. As will be shown in the next section, the effective neutrino couplings are induced in the case of $Z^{\prime}$ portal with a gauge kinetic mixing, but there is no bound on them other than the lifetime bound from the age of the Universe.

For $m_{e}, m_{\chi_{1}}, m_{Z^{\prime}} \gg q \sim m_{e} \Delta m, m_{e} \ll m_{\chi_{1}}$, and $\Delta m \ll m_{\chi_{1}}$, the total scattering cross section for $\chi_{1} e \rightarrow \chi_{2} e$, up to the phase space factor $P^{2}(v)$ in eq. (2.25), is given by

$$
\begin{aligned}
\bar{\sigma}_{e} & \simeq \frac{v_{\chi}^{2} v_{e}^{2} g_{Z^{\prime}}^{4} \mu_{1}^{2}}{\pi m_{Z^{\prime}}^{4}} \\
& \simeq\left(\frac{v_{\chi} g_{Z^{\prime}}}{0.6}\right)^{2}\left(\frac{v_{e} g_{Z^{\prime}}}{10^{-4} e}\right)^{2}\left(\frac{1 \mathrm{GeV}}{m_{Z^{\prime}}}\right)^{4}\left(\frac{\mu_{1}}{m_{e}}\right)^{2} \times 10^{-44} \mathrm{~cm}^{2}
\end{aligned}
$$

where $e$ is the electromagnetic coupling. Thus, we need to have nonzero vector couplings to both dark matter and electron for the scattering cross section without velocity suppression. In order to explain the XENON1T electron recoil events near $E_{R}=2-3 \mathrm{keV}$ in our model, we take $\Delta m \simeq 2.5 \mathrm{keV}$ and the required scattering cross section gives rise to the following useful formula,

$$
\left(\frac{v_{\chi} g_{Z^{\prime}}}{0.6}\right)^{2}\left(\frac{v_{e} g_{Z^{\prime}}}{10^{-4} e}\right)^{2}\left(\frac{1 \mathrm{GeV}}{m_{Z^{\prime}}}\right)^{4}\left(\frac{0.3 \mathrm{GeV}}{m_{\chi_{1}}}\right)\left(\frac{\Omega_{\mathrm{DM}}}{\Omega_{\chi_{1}}}\right) \simeq 1
$$

where $\Omega_{\mathrm{DM}}$ is the observed total abundance of dark matter and $\Omega_{\chi_{1}}$ is the abundance of the dark fermion $\chi_{1}$. Therefore, light dark matter and $Z^{\prime}$ mediator are favored by the explanation of the Xenon excess with exothermic dark matter. As we scale up the $Z^{\prime}$ gauge coupling, we can take a larger value of $m_{Z^{\prime}}^{4} m_{\chi_{1}}$ in order to maintain the number of the electron recoil events.

We remark on the choices of the $Z^{\prime}$ couplings in view of the Xenon electron excess. First, we took the $Z^{\prime}$ couplings to be consistent with the dilepton bounds from BaBar, $\left|v_{e}\right| g_{Z^{\prime}} \lesssim 10^{-4} e$ for $0.02 \mathrm{GeV}<m_{Z^{\prime}}<10.2 \mathrm{GeV}$ [43], or the bound from mono-photon + MET from BaBar [44], $\left|v_{e}\right| g_{Z^{\prime}} \lesssim\left(4 \times 10^{-4}-10^{-3}\right) e$ for $m_{Z^{\prime}}<8 \mathrm{GeV}$. There are other bounds from beam dump experiments [45-48] that limit $\left|v_{e}\right| g_{Z^{\prime}}$ at the level of $10^{-3}$ or stronger for $m_{Z^{\prime}} \lesssim 0.1 \mathrm{GeV}$ than in BaBar experiment. In the parameter space of our interest, we focus on $m_{Z^{\prime}} \gtrsim 0.1 \mathrm{GeV}$, for which the BaBar bounds are most stringent at present. Thus, we take into account only the BaBar bounds in the later discussion on phenomenological constants in the next subsection and the next section on microscopic models.

\subsection{Dark matter annihilation and relic density}

Dark matter fermions $\chi_{1}$ and $\chi_{2}$ can co-annihilate into a pair of electrons as well as into a pair of $Z^{\prime}$ gauge bosons if kinematically allowed. Then, taking $\Delta m \ll m_{\chi_{1}}$ and ignoring the lepton masses, the total annihilation cross section for $n_{\mathrm{DM}}=n_{\chi_{1}}+n_{\chi_{2}}$ with $\chi_{1} \bar{\chi}_{2} \rightarrow e \bar{e}, \nu \bar{\nu}$ and $\chi_{1} \bar{\chi}_{1}, \chi_{2} \bar{\chi}_{2} \rightarrow Z^{\prime} Z^{\prime}$ is given by

$$
\langle\sigma v\rangle=\frac{1}{2}\langle\sigma v\rangle_{\chi_{1} \bar{\chi}_{2} \rightarrow e \bar{e}, \nu \bar{\nu}}+\frac{1}{2}\langle\sigma v\rangle_{\chi_{1} \bar{\chi}_{2} \rightarrow Z^{\prime} Z^{\prime}}
$$


with

$$
\begin{aligned}
\langle\sigma v\rangle_{\chi_{1} \bar{\chi}_{2} \rightarrow e \bar{e}, \nu \bar{\nu}} & =\frac{g_{Z^{\prime}}^{4} v_{\chi}^{2}}{\pi}\left[v_{e}^{2}+a_{e}^{2}+N_{\nu}\left(v_{\nu}^{2}+a_{\nu}^{2}\right)\right] \frac{m_{\chi_{1}}^{2}}{\left(m_{Z^{\prime}}^{2}-4 m_{\chi_{1}}^{2}\right)^{2}+\Gamma_{Z^{\prime}}^{2} m_{Z^{\prime}}^{2}}, \\
\langle\sigma v\rangle_{\chi_{1} \bar{\chi}_{1}, \chi_{2} \bar{\chi}_{2} \rightarrow Z^{\prime} Z^{\prime}} & =\frac{g_{Z^{\prime}}^{4}}{4 \pi}\left[v_{\chi}^{4}+a_{\chi}^{4}+2 v_{\chi}^{2} a_{\chi}^{2}\left(4 \frac{m_{\chi_{1}}^{2}}{m_{Z^{\prime}}^{2}}-3\right)\right] \frac{m_{\chi_{1}}^{2}}{\left(m_{Z^{\prime}}^{2}-2 m_{\chi_{1}}^{2}\right)^{2}}\left(1-\frac{m_{Z^{\prime}}^{2}}{m_{\chi_{1}}^{2}}\right)^{3 / 2} .
\end{aligned}
$$

We note that the contributions coming from $a_{\chi}$ to the annihilation cross section are $p$-wave suppressed. Since we need $v_{\chi} \neq 0$ for explaining the Xenon electron excess, the $p$-wave annihilations are sub-dominant.

For light dark matter with sub-GeV mass, once $\chi_{1} \bar{\chi}_{1}, \chi_{2} \bar{\chi}_{2} \rightarrow Z^{\prime} Z^{\prime}$ is open, the resultant annihilation cross section would be too large for a sizable $g_{Z^{\prime}}$ to account for the correct relic density. Thus, in this case, we can take $m_{\chi_{1}}<m_{Z^{\prime}}$ such that the annihilation of the dark matter fermion $\chi_{1}$ into a pair of $Z^{\prime}$ is forbidden at zero temperature, but it is open in the tail of the Boltzmann distribution at a finite temperature during freeze-out [2933]. Then, from the detailed balance condition for the forbidden channels, the effective annihilation cross section for the forbidden channels, $\chi_{1} \bar{\chi}_{1}, \chi_{2} \bar{\chi}_{2} \rightarrow Z^{\prime} Z^{\prime}$ becomes

$$
\langle\sigma v\rangle_{\chi_{1} \bar{\chi}_{1}, \chi_{2} \bar{\chi}_{2} \rightarrow Z^{\prime} Z^{\prime}}=\frac{\left(n_{Z^{\prime}}^{\mathrm{eq}}\right)^{2}}{n_{\chi_{1}}^{\mathrm{eq}} n_{\chi_{2}}^{\mathrm{eq}}}\langle\sigma v\rangle_{Z^{\prime} Z^{\prime} \rightarrow \chi_{1} \bar{\chi}_{1}, \chi_{2} \bar{\chi}_{2}}
$$

with

$$
\begin{aligned}
\langle\sigma v\rangle_{Z^{\prime} Z^{\prime} \rightarrow \chi_{1} \bar{\chi}_{1}, \chi_{2} \bar{\chi}_{2}}= & \frac{4 g_{Z^{\prime}}^{4}}{9 \pi m_{Z^{\prime}}^{2}}\left[v_{\chi}^{4}+a_{\chi}^{4}\left(\frac{m_{Z^{\prime}}^{2}+2 m_{\chi_{1}}^{2}}{m_{Z^{\prime}}^{2}+m_{\chi_{1}}^{2}}\right)+2 v_{\chi}^{2} a_{\chi}^{2}\left(\frac{3 m_{Z^{\prime}}^{2}-2 m_{\chi_{1}}^{2}}{m_{Z^{\prime}}^{2}+m_{\chi_{1}}^{2}}\right)\right] \\
& \times\left(1+\frac{m_{\chi_{1}}^{2}}{m_{Z^{\prime}}^{2}}\right)\left(1-\frac{m_{\chi_{1}}^{2}}{m_{Z^{\prime}}^{2}}\right)^{3 / 2} .
\end{aligned}
$$

Here, for $\Delta m \ll m_{\chi_{1}}$, the Boltzmann suppression factor can be approximated to

$$
\frac{\left(n_{Z^{\prime}}^{\mathrm{eq}}\right)^{2}}{n_{\chi 1}^{\mathrm{eq}} n_{\chi_{2}}^{\mathrm{eq}}} \simeq\left(\frac{n_{Z^{\prime}}^{\mathrm{eq}}}{n_{\chi_{1}}^{\mathrm{eq}}}\right)^{2} \simeq \frac{9}{4}\left(\frac{m_{Z^{\prime}}}{m_{\chi_{1}}}\right)^{3} e^{-2\left(m_{Z^{\prime}}-m_{\chi_{1}}\right) / T}
$$

The forbidden channels are important for obtaining the correct relic density for light dark matter, because the strong annihilation cross section can be compensated by the Boltzmann suppression factor [29-33].

Furthermore, for $m_{\chi_{1}}>m_{\chi_{2}}$, we have additional annihilation channels active even at zero temperature, $\chi_{1} \chi_{1} \rightarrow \chi_{2} \chi_{2}, \chi_{1} \bar{\chi}_{1} \rightarrow \chi_{2} \bar{\chi}_{2}$, and their complex conjugates. Then, the additional annihilation cross sections for $\chi_{1}$ are given by

$$
\begin{aligned}
& \langle\sigma v\rangle_{\chi_{1} \chi_{1} \rightarrow \chi_{2} \chi_{2}}=\frac{\sqrt{2}}{16 \pi} \frac{g_{Z^{\prime}}^{4} m_{\chi_{1}}^{2}}{m_{Z^{\prime}}^{4}} \sqrt{\frac{\Delta m}{m_{\chi_{1}}}}\left(v_{\chi}^{4}+6 a_{\chi}^{2} v_{\chi}^{2}+9 a_{\chi}^{4}\right), \\
& \langle\sigma v\rangle_{\chi_{1} \bar{\chi}_{1} \rightarrow \chi_{2} \bar{\chi}_{2}}=\frac{\sqrt{2}}{8 \pi} \frac{g_{Z^{\prime}}^{4} m_{\chi_{1}}^{2}}{m_{Z^{\prime}}^{4}} \sqrt{\frac{\Delta m}{m_{\chi_{1}}}}\left(v_{\chi}^{4}+3 a_{\chi}^{4}\right) .
\end{aligned}
$$


For $T \gtrsim \Delta m=2.5 \mathrm{keV}$, the inverse annihilation processes, $\chi_{2} \chi_{2} \rightarrow \chi_{1} \chi_{1}, \chi_{2} \bar{\chi}_{2} \rightarrow \chi_{1} \bar{\chi}_{1}$, and their complex conjugates, occur as often as the above annihilation, so the additional annihilation processes have no impact on the total dark matter density at the time of freeze-out of the annihilation processes, given in eq. (3.9). However, the $\chi_{1}$ component could keep annihilating and its abundance would be Boltzmann suppressed by $e^{-\Delta / T_{\chi_{1}}}$ with $T_{\chi_{1}}=T^{2} / T_{\mathrm{kd}}$ where $T_{\mathrm{kd}}$ is the kinetic decoupling temperature, which is the smaller of the decoupling temperature of dark matter and the electron decoupling temperature $\sim 1 \mathrm{MeV}$. Then, in order to avoid the Boltzmann suppression for the relic abundance of the heavier component, we need to impose

$$
T_{\chi_{1}} \gtrsim \Delta m
$$

which corresponds to the bound on the radiation temperature,

$$
T \gtrsim \sqrt{T_{\mathrm{kd}} \Delta m}
$$

The decoupling temperature for the $2 \rightarrow 2$ annihilation processes is determined by

$$
n_{\chi_{1}} \max \left(\langle\sigma v\rangle_{\chi_{1} \chi_{1} \rightarrow \chi_{2} \chi_{2}},\langle\sigma v\rangle_{\chi_{1} \bar{\chi}_{1} \rightarrow \chi_{2} \bar{\chi}_{2}}\right)=H .
$$

Thus, imposing the above equation with eq. (3.17) and for $\left|v_{\chi}\right|=\left|a_{\chi}\right|$, we find the upper limit on the $Z^{\prime}$ gauge coupling as follows,

$$
\frac{\left|v_{\chi}\right| g_{Z^{\prime}} m_{\chi_{1}}}{m_{Z^{\prime}}} \lesssim 0.035\left(\frac{\Omega_{\mathrm{DM}} / 2}{\Omega_{\chi_{1}}}\right)^{1 / 4}\left(\frac{m_{\chi_{1}}}{100 \mathrm{MeV}}\right)^{1 / 2}\left(\frac{m_{\chi_{1}} / \Delta m}{4 \times 10^{4}}\right)^{3 / 8}\left(\frac{\Delta m /(2.5 \mathrm{keV})}{T_{\mathrm{kd}} /(10 \mathrm{MeV})}\right)^{1 / 8}
$$

Therefore, we need to impose the above condition that the annihilation processes, $\chi_{1} \chi_{1} \rightarrow$ $\chi_{2} \chi_{2}, \chi_{1} \bar{\chi}_{1} \rightarrow \chi_{2} \bar{\chi}_{2}$, decouple sufficiently early at $T_{\chi_{1}} \gtrsim \Delta m$, in the following discussion on the relic density.

As a consequence, the dark matter number density is given by $n_{\mathrm{DM}}=n_{\chi_{1}}+n_{\chi_{2}}$ with $n_{\chi_{1}} \simeq n_{\chi_{2}}$ for the early decoupling of the $2 \rightarrow 2$ annihilations, so the corresponding relic abundance is determined as

$$
\Omega_{\mathrm{DM}} h^{2}=0.12\left(\frac{10.75}{g_{*}\left(T_{f}\right)}\right)^{1 / 2}\left(\frac{x_{f}}{20}\right)\left(\frac{4.3 \times 10^{-9} \mathrm{GeV}^{-2}}{x_{f} \int_{x_{f}}^{\infty} x^{-2}\langle\sigma v\rangle}\right)
$$

where $\langle\sigma v\rangle$ is given by eq. (3.9) and $x_{f}=m_{\chi_{1}} / T_{f}$ at freeze-out temperature. Therefore, parametrizing the effective annihilation cross section by $\langle\sigma v\rangle=\frac{\alpha_{\text {eff }}^{2}}{m_{\chi_{1}}^{2}}$, we can achieve a correct relic density, provided that

$$
m_{\chi_{1}} \simeq 150 \mathrm{MeV}\left(\frac{\alpha_{\mathrm{eff}}}{10^{-5}}\right)
$$

As a result, we can get the correct relic density by taking a small effective coupling $\alpha_{\text {eff }}$ from small SM couplings, $v_{e}, v_{\nu}$, or due to the Boltzmann-suppression, $\alpha_{\text {eff }} \sim g_{Z^{\prime}}^{2} e^{-x_{f}\left(m_{Z^{\prime}}-m_{\chi_{1}}\right)}$, for $g_{Z^{\prime}}=0.6$ and $\frac{m_{Z^{\prime}}}{m_{\chi_{1}}}-1 \simeq 0.4$, being consistent with the constraint from the Xenon excess with $\Omega_{\chi_{1}} \simeq \frac{1}{2} \Omega_{\mathrm{DM}}$ in eq. (3.8). 

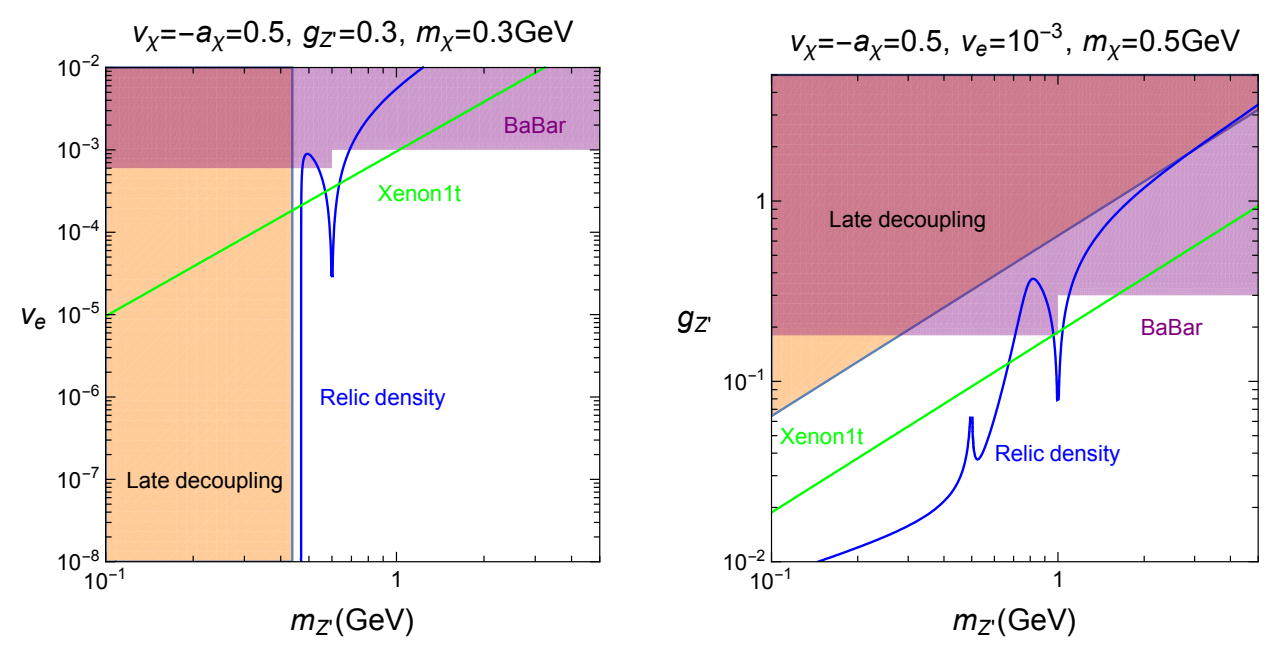

Figure 2. Parameter space for relic density and XENON1T electron excess. The favored region by XENON1T electron excess is along the green line, the relic density is satisfied along the blue line. The purple region on the left plot is excluded by the BaBar limits on the visible and invisible decays of $Z^{\prime}$. We have taken $v_{\chi}=-a_{\chi}=0.5, a_{e}=0$, and $m_{\chi}=0.3,0.5 \mathrm{GeV}$ on left and right plots, respectively, and $g_{Z^{\prime}}=0.3$ on left and $v_{e}=10^{-3}$ on right. In the orange region, the relic density for the heavier state is Boltzmann-suppressed for $T_{\mathrm{kd}}=1 \mathrm{MeV}$.

In figure 2, we show the parameter space for relic density in comparison to Xenon electron excess, for $m_{Z^{\prime}}$ vs $v_{e}$ on left and $m_{Z^{\prime}}$ vs $g_{Z^{\prime}}$ on right. We have fixed $v_{\chi}=-a_{\chi}=0.5$, $a_{e}=0$, and $m_{\chi}=0.3,0.5 \mathrm{GeV}$ on left and right plots, respectively, and $g_{Z^{\prime}}=0.3$ on left and $v_{e}=10^{-3}$ on right. We have imposed the BaBar bounds from visible and invisible decays of $Z^{\prime}$ on the effective electron coupling $v_{e}$. The correct relic density is saturated along the blue lines, showing that there is a parameter space compatible with the Xenon electron excess in green lines and the BaBar bounds as well as the late decoupling in orange region.

In the above discussion, we focused on the annihilation channels in the minimal scenario for the Xenon electron excess. However, if the aforementioned annihilation channels with $Z^{\prime}$ interactions are not sufficient for a correct relic density, due to small $Z^{\prime}$ couplings to the $\mathrm{SM}$, we can also consider the dark matter self-interactions for dark matter annihilation, in particular, as in the case of SIMP dark matter [49-55] where sub-GeV light dark matter is a natural outcome of the $3 \rightarrow 2$ annihilations with strong self-interactions. In this case, the relic density condition on the visible coupling becomes weaker, so there is more parameter opening up for the Xenon electron excess.

\section{Microscopic models}

In this section, we propose a microscopic model for exothermic dark matter by taking two left-handed dark fermions, $\psi_{1}$ and $\psi_{2}$, with opposite charges, +1 and -1 , under the dark $\mathrm{U}(1)^{\prime}$ symmetry. We also introduce a dark Higgs $\phi$ with charge -2 under the $\mathrm{U}(1)^{\prime}$. We assume that all the SM particles are neutral under the $\mathrm{U}(1)^{\prime}$, but dark matter can communicate with the SM through 1) the gauge kinetic mixing, $\sin \xi$, or 2) the mixing between 
electron and an extra vector-like lepton. We first discuss the dark matter interactions and proceed to derive the effective interactions for the electron in each case of portal models.

The Lagrangian for the dark sector is given, as follows,

$$
\begin{aligned}
\mathcal{L}= & -\frac{1}{4} F_{\mu \nu}^{\prime} F^{\prime \mu \nu}+\left|D_{\mu} \phi\right|^{2}-V(\phi, H) \\
& +i \bar{\psi}_{1 L} \gamma^{\mu} D_{\mu} \psi_{1 L}+i \bar{\psi}_{2 L} \gamma^{\mu} D_{\mu} \psi_{2 L} \\
& -m_{\psi} \psi_{1} \psi_{2}-y_{1} \phi \psi_{1} \psi_{1}-y_{2} \phi^{*} \psi_{2} \psi_{2}+\text { h.c. }
\end{aligned}
$$

where $F_{\mu \nu}^{\prime}=\partial_{\mu} Z_{\nu}^{\prime}-\partial_{\nu} Z_{\mu}^{\prime}, B_{\mu \nu}$ is the field strength tensor for the SM hypercharge, the covariant derivatives are $D_{\mu} \phi=\left(\partial_{\mu}+2 i g_{Z^{\prime}} Z_{\mu}^{\prime}\right) \phi, D_{\mu} \psi_{1 L}=\left(\partial_{\mu}-i g_{Z^{\prime}} Z_{\mu}^{\prime}\right) \psi_{1 L}, D_{\mu} \psi_{2 L}=$ $\left(\partial_{\mu}+i g_{Z^{\prime}} Z_{\mu}^{\prime}\right) \psi_{2 L}, m_{\psi}$ is the Dirac mass for dark fermions, and $y_{1,2}$ are the Yukawa couplings for dark fermions. Here, $V(\phi, H)$ is the scalar potential for the singlet scalar $\phi$ and the SM Higgs.

After the dark Higgs gets a VEV as $\langle\phi\rangle=v_{\phi}$, the $Z^{\prime}$ gauge boson receives mass $m_{Z^{\prime}}=2 \sqrt{2} g_{Z^{\prime}} v_{\phi}$, and there appears a mass mixing between $\psi_{1}$ and $\psi_{2}$. Then, diagonalizing the mass matrix for the dark fermions, we get the mass eigenvalues and the mixing matrix, as follows,

$$
m_{\chi_{1,2}}^{2}=m_{\psi}^{2}+2\left(y_{1}^{2}+y_{2}^{2}\right) v_{\phi}^{2} \pm 2 \sqrt{\left(y_{1}^{2}-y_{2}^{2}\right)^{2} v_{\phi}^{4}+\left(y_{1}+y_{2}\right)^{2} v_{\phi}^{2} m_{\psi}^{2}},
$$

and

$$
\left(\begin{array}{l}
\chi_{1} \\
\chi_{2}
\end{array}\right)=\left(\begin{array}{cc}
\cos \theta & -\sin \theta \\
\sin \theta & \cos \theta
\end{array}\right)\left(\begin{array}{l}
\psi_{2} \\
\psi_{1}
\end{array}\right)
$$

with

$$
\sin 2 \theta=-\frac{4\left(y_{1}+y_{2}\right) v_{\phi} m_{\psi}}{m_{\chi_{2}}^{2}-m_{\chi_{1}}^{2}}
$$

For simplicity, we take $y_{1}=y_{2}$, then the mass eigenvalues become $m_{\chi_{1,2}}=m_{\psi} \pm 2 y_{1} v_{\phi}$ and the mixing angle is given by $\theta=\frac{\pi}{4}$. Then, the mass difference can be small as far as $2\left|y_{1}\right| v_{\phi} \ll m_{\psi}$. There was a recent discussion on the microscopic model for exothermic dark matter with a complex scalar field where the breaking of dark $\mathrm{U}(1)^{\prime}$ makes a small mass splitting between the real scalar fields [5].

For instance, for $m_{\psi} \sim 1 \mathrm{GeV}$, we need $y_{1} \sim 1.5 \times 10^{-6}$. As a result, including the $Z^{\prime}$-portal couplings to the SM fermions for a small gauge kinetic mixing, we summarize the $Z^{\prime}$ gauge interactions as follows,

$$
\mathcal{L}_{\mathrm{DM}}=-g_{Z^{\prime}} Z_{\mu}^{\prime}\left(\bar{\chi}_{1} \gamma^{\mu} P_{L} \chi_{2}+\bar{\chi}_{2} \gamma^{\mu} P_{L} \chi_{1}\right)
$$

Then, we obtain the effective dark matter couplings in the Lagrangian (3.1) as

$$
v_{\chi}=-a_{\chi}=-\frac{1}{2}
$$

As a result, we can realize the transition interactions between two states of dark matter via the $Z^{\prime}$ mediator, that are necessary for explaining the Xenon excess. 

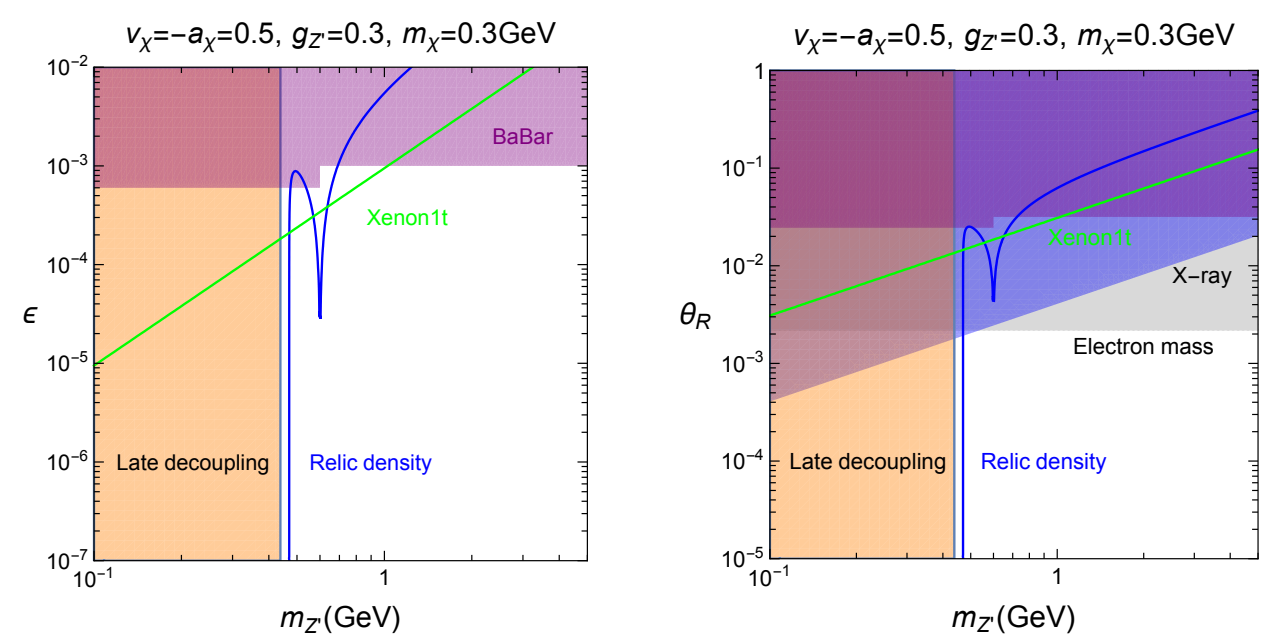

Figure 3. Parameter space for relic density and XENON1T electron excess in microscopic models. (Left) $Z^{\prime}$ portal: we have taken $v_{\chi}=-a_{\chi}=0.5, g_{Z^{\prime}}=0.3$ and $m_{\chi}=0.3 \mathrm{GeV}$. The color notations are the same as those in figure 2. (Right) Vector-like lepton portal: we have taken $v_{\chi}=-a_{\chi}=0.5$, $g_{Z^{\prime}}=0.3$ and $m_{\chi}=0.3 \mathrm{GeV}$. The red region is excluded by the X-ray searches and the gray region is disfavored by the electron mass. In the orange region, the relic density for the heavier state is Boltzmann-suppressed for $T_{\mathrm{kd}}=1 \mathrm{MeV}$.

\section{$4.1 \quad Z^{\prime}$-portal}

In the presence of a gauge kinetic mixing,

$$
\mathcal{L}_{\text {kin }- \text { mix }}=-\frac{1}{2} \sin \xi B_{\mu \nu} F^{\prime \mu \nu},
$$

the mixing between $Z^{\prime}$ and $Z$ gauge bosons gives rise to the $Z^{\prime}$ gauge interactions to the $\mathrm{SM}$ as

$$
\mathcal{L}_{\text {eff }, \mathrm{I}}=-e \varepsilon Z_{\mu}^{\prime}\left(\bar{e} \gamma^{\mu} e+\frac{m_{Z^{\prime}}^{2}}{2 c_{W}^{2} m_{Z}^{2}} \bar{\nu} \gamma^{\mu} P_{L} \nu\right)+\cdots
$$

where $\varepsilon \equiv \xi \cos \theta_{W}$ and the ellipse denotes the electromagnetic and neutral current interactions for the rest of the SM fermions. Therefore, there are not only electron couplings but also neutrino couplings, although the latter being further suppressed by $m_{Z^{\prime}}^{2} / m_{Z}^{2}[56]$. Consequently, we can identify the effective couplings in the Lagrangian (3.1), as follows,

$$
v_{e}=-\frac{e \varepsilon}{g_{Z^{\prime}}}, \quad a_{e}=0, \quad v_{\nu}=-a_{\nu}=-\frac{e \varepsilon m_{Z^{\prime}}^{2}}{4 c_{W}^{2} g_{Z^{\prime}} m_{Z}^{2}} .
$$

Then, the lifetime of the dark fermion $\chi_{1}$ is given by

$$
\tau_{\chi_{1}}=\frac{1}{\Gamma\left(\chi_{1} \rightarrow \chi_{2} \nu \bar{\nu}\right)}=\left(\frac{10^{-4} e}{\varepsilon g_{Z^{\prime}}}\right)^{2}\left(\frac{2.5 \mathrm{keV}}{\Delta m}\right)^{5} 8.9 \times 10^{24} \mathrm{sec} .
$$

Therefore, the dark fermion $\chi_{1}$ is much long-lived than the age of the Universe, so it can be responsible for the Xenon electron excess through the inelastic scattering, as discussed in the previous section. 
On the left of figure (3), we show the parameter space in $\varepsilon$ vs $m_{Z^{\prime}}$, that is consistent with the Xenon electron excess, the correct relic density as well as the BaBar bounds. We have taken $v_{\chi}=-a_{\chi}=0.5, g_{Z^{\prime}}=0.3$ and $m_{\chi} \equiv m_{\chi_{1}}=0.3 \mathrm{GeV}$. Since the effective axial coupling $a_{e}$ vanishes, there is no bound from the X-ray searches. In the orange region, the relic density for the heavier state is Boltzmann-suppressed due to the $2 \rightarrow 2$ self-annihilations. As a result, there is a consistent parameter space for the Xenon electron excess, which can be probed in the future experiments.

\subsection{Vector-like lepton portal}

We introduce an extra vector-like charged lepton $E$ which has charge -2 under the $\mathrm{U}(1)^{\prime}$ but is singlet under the $\mathrm{SU}(2)_{L}$. Then, the mixing between the SM right-handed electron and the vector-like lepton is given by

$$
\mathcal{L}_{\mathrm{VL}}=-M_{E} \bar{E} E-\left(y_{E} \phi \bar{E} e_{R}+\text { h.c. }\right)
$$

As a consequence, the mass matrix for the electron and the vector-like lepton takes

$$
M_{e}=\left(\begin{array}{cc}
m_{e} & 0 \\
y_{E} v_{\phi} & M_{E}
\end{array}\right) .
$$

Then, the mass eigenvalues are given by

$$
m_{f_{1,2}}^{2}=\frac{1}{2}\left(m_{e}^{2}+M_{E}^{2}+y_{E}^{2} v_{\phi}^{2} \mp \sqrt{\left(m_{e}^{2}+y_{E}^{2} v_{\phi}^{2}-M_{E}^{2}\right)^{2}+4 y_{E}^{2} v_{\phi}^{2} M_{E}^{2}}\right) .
$$

On the other hand, the mixing angles for the right-handed electrons and the left-handed electrons are given [57], respectively, by

$$
\begin{aligned}
& \sin \left(2 \theta_{R}\right)=-\frac{2 y_{E} v_{\phi} M_{E}}{m_{f_{1}}^{2}-m_{f_{2}}^{2}}, \\
& \sin \left(2 \theta_{L}\right)=\frac{m_{e}^{2}}{m_{f_{1}} m_{f_{2}}} \sin \left(2 \theta_{R}\right) .
\end{aligned}
$$

Therefore, for $m_{e}, y_{E} v_{\phi} \ll M_{E}$ and $\left(y_{E} v_{\phi} / M_{E}\right)^{2} \lesssim\left(m_{e} / M_{E}\right)$, the mass eigenvalues are approximated to $m_{f_{1}} \sim m_{e}$ and $m_{f_{2}} \sim M_{E}$, and the mixing angles become $\theta_{R} \sim \frac{2 y_{E} v_{\phi}}{M_{E}}$ and $\theta_{L} \sim \frac{m_{e}}{M_{E}} \theta_{R}$. Given the experimental bound on the vector-like charged lepton from LEP and LHC, $M_{E} \gtrsim 100 \mathrm{GeV}$, we have $\frac{m_{e}}{M_{E}} \lesssim 5 \times 10^{-6}$, so we can ignore the mixing for the left-handed electrons. But, for $m_{f_{1}} \sim m_{e}$, the mixing angle for the right-handed electrons is bounded by $\theta_{R} \lesssim \sqrt{\frac{m_{e}}{M_{E}}}$, thus $\theta_{R}$ can be as large as $2.2 \times 10^{-3}$. On the other hand, we note that the suppressed mixing angle $\theta_{L}$ for the left-handed electron is consistent with electroweak precision data.

Consequently, we get the following effective interactions for $Z^{\prime}$,

$$
\begin{aligned}
\mathcal{L}_{\mathrm{eff}, \mathrm{II}}=-2 g_{Z^{\prime}} Z_{\mu}^{\prime}\left(\bar{E} \gamma^{\mu} E+\theta_{R}^{2} \bar{e} \gamma^{\mu} P_{R} e-\theta_{R} \bar{E} \gamma^{\mu} P_{R} e-\theta_{R} \bar{e} \gamma^{\mu} P_{R} E\right. \\
\left.+\theta_{L}^{2} \bar{e} \gamma^{\mu} P_{L} e-\theta_{L} \bar{E} \gamma^{\mu} P_{L} e-\theta_{L} \bar{e} \gamma^{\mu} P_{L} E\right)
\end{aligned}
$$




$$
\begin{aligned}
& -\frac{g}{2 c_{W}} Z_{\mu}\left(\bar{e}_{L} \gamma^{\mu} P_{L} e+\theta_{L} \bar{E} \gamma^{\mu} P_{L} e+\theta_{L} \bar{e} \gamma^{\mu} P_{L} E+\theta_{L}^{2} \bar{E} \gamma^{\mu} P_{L} E\right) \\
& -\frac{g}{\sqrt{2}} \theta_{L} \bar{E} \gamma^{\mu} P_{L} \nu W_{\mu}^{-}+\text {h.c. }
\end{aligned}
$$

Here, we used the same notations for mass eigenstates as for interaction eigenstates, electron-like and vector-like. Therefore, we can identify the effective couplings in the Lagrangian (3.1), as follows,

$$
v_{e}=a_{e}=-\theta_{R}^{2}, \quad v_{\nu}=a_{\nu}=0 .
$$

As a result, we obtain the necessary electron coupling to $Z^{\prime}$ for explaining the Xenon excess through the small mixing between the SM right-handed electron and the vector-like lepton. In the model with vector-like lepton portal, however, there is no direct coupling between $Z^{\prime}$ and neutrinos. Even the $Z^{\prime}-e-E$ vertex with $E$ decaying into the SM particles does not make the dark fermion $\chi_{1}$ to decay, provided that $\Delta m<2 m_{e}$ is chosen.

On the right of figure (3), we show the parameter space in $\theta_{R}$ vs $m_{Z^{\prime}}$, that is consistent with the Xenon electron excess, the correct relic density as well as the BaBar bounds. We have taken $v_{\chi}=-a_{\chi}=0.5, g_{Z^{\prime}}=0.3$ and $m_{\chi}=m_{\chi_{1}}=0.3 \mathrm{GeV}$. Since the effective axial coupling $a_{e}$ is nonzero, the bound from the X-ray searches in gray excludes the relic density condition in blue line, and the consistent parameter space is pushed towards a larger $g_{Z^{\prime}}$ or the resonance region near $m_{Z^{\prime}} \sim 2 m_{\chi}$. We note that the gray region is disfavored by the electron mass. We also note that in the orange region, the relic density for the heavier state is Boltzmann-suppressed due to the $2 \rightarrow 2$ self-annihilations.

\section{Conclusions}

We proposed exothermic dark matter to explain the recent electron excess reported by XENON1T experiment. Even for a small dark matter velocity, as known from the standard Maxwellian velocity distribution of dark matter, we achieved the appropriate electron recoil energy at about $2.5 \mathrm{keV}$ by considering the down-scattering of the heavier dark matter state off the electron into the lighter state. Thus, we showed that about 50 recoil events over $E_{R}=2-3 \mathrm{keV}$, which are most significant, can be explained in this scenario up to the detector resolution.

Including the effects of the phase-space enhancement and the atomic excitation factor, we derived the required scattering cross section for the Xenon excess to be about $\sigma_{e} \sim 10^{-44} \mathrm{~cm}^{2}$ for sub-GeV light dark matter. We took the effective theory approach for exothermic dark matter with a massive $Z^{\prime}$ mediator and discussed the implications of the Xenon excess for dark matter interactions to $Z^{\prime}$ and the dark matter relic density. We also provided microscopic models with $Z^{\prime}$ portal and vector-like portal, realizing the required dark matter and electron couplings to $Z^{\prime}$, while the heavier state of dark matter is long-lived enough to satisfy the bounds from the X-ray or the neutrino experiments.

\section{Acknowledgments}

I would like to thank the CERN Theory group members for the brain-storming discussion on the Xenon excess during the virtual coffee meeting, Juri Smirnov, Jure Zupan and Bin 
Zhu for communications on the interpretation of the Xenon excess, and Yoo-Jin Kang and Soonbin Kim for checking the formulas for the electron recoil energy in the paper. In particular, I appreciate the comment from Jure Zupan to check the two-photon decay mode of the heavier state of dark matter in more detail. The work is supported in part by Basic Science Research Program through the National Research Foundation of Korea (NRF) funded by the Ministry of Education, Science and Technology (NRF-2019R1A2C2003738 and NRF-2018R1A4A1025334).

Open Access. This article is distributed under the terms of the Creative Commons Attribution License (CC-BY 4.0), which permits any use, distribution and reproduction in any medium, provided the original author(s) and source are credited.

\section{References}

[1] XENON collaboration, Excess electronic recoil events in XENON1T, Phys. Rev. D 102 (2020) 072004 [arXiv: 2006.09721] [inSPIRE].

[2] F. Takahashi, M. Yamada and W. Yin, XENON1T excess from anomaly-free axionlike dark matter and its implications for stellar cooling anomaly, Phys. Rev. Lett. 125 (2020) 161801 [arXiv: 2006. 10035] [INSPIRE].

[3] K. Kannike, M. Raidal, H. Veermäe, A. Strumia and D. Teresi, Dark matter and the XENON1T electron recoil excess, Phys. Rev. D 102 (2020) 095002 [arXiv:2006.10735] [INSPIRE].

[4] G. Alonso-Álvarez, F. Ertas, J. Jaeckel, F. Kahlhoefer and L.J. Thormaehlen, Hidden photon dark matter in the light of XENON1T and stellar cooling, JCAP 11 (2020) 029 [arXiv: 2006.11243] [INSPIRE].

[5] K. Harigaya, Y. Nakai and M. Suzuki, Inelastic dark matter electron scattering and the XENON1T excess, Phys. Lett. B 809 (2020) 135729 [arXiv:2006.11938] [INSPIRE].

[6] B. Fornal, P. Sandick, J. Shu, M. Su and Y. Zhao, Boosted dark matter interpretation of the XENON1T excess, Phys. Rev. Lett. 125 (2020) 161804 [arXiv:2006.11264] [INSPIRE].

[7] C. Boehm, D.G. Cerdeno, M. Fairbairn, P.A.N. Machado and A.C. Vincent, Light new physics in XENON1T, Phys. Rev. D 102 (2020) 115013 [arXiv:2006.11250] [INSPIRE].

[8] L. Su, W. Wang, L. Wu, J.M. Yang and B. Zhu, Atmospheric dark matter and Xenon1T excess, Phys. Rev. D 102 (2020) 115028 [arXiv:2006.11837] [InSPIRE].

[9] M. Du, J. Liang, Z. Liu, V.Q. Tran and Y. Xue, On-shell mediator dark matter models and the Xenon1T anomaly, arXiv:2006.11949 [INSPIRE].

[10] L. Di Luzio, M. Fedele, M. Giannotti, F. Mescia and E. Nardi, Solar axions cannot explain the XENON1T excess, Phys. Rev. Lett. 125 (2020) 131804 [arXiv:2006.12487] [InSPIRE].

[11] N.F. Bell, J.B. Dent, B. Dutta, S. Ghosh, J. Kumar and J.L. Newstead, Explaining the XENON1T excess with Luminous Dark Matter, Phys. Rev. Lett. 125 (2020) 161803 [arXiv:2006.12461] [INSPIRE].

[12] Y. Chen, M.-Y. Cui, J. Shu, X. Xue, G. Yuan and Q. Yuan, Sun heated MeV-scale dark matter and the XENON1T electron recoil excess, arXiv:2006.12447 [INSPIRE]. 
[13] D. Aristizabal Sierra, V. De Romeri, L.J. Flores and D.K. Papoulias, Light vector mediators facing XENON1T data, Phys. Lett. B 809 (2020) 135681 [arXiv:2006.12457] [INSPIRE].

[14] J. Buch, M.A. Buen-Abad, J. Fan and J.S.C. Leung, Galactic origin of relativistic bosons and XENON1T excess, JCAP 10 (2020) 051 [arXiv: 2006.12488] [INSPIRE].

[15] G. Choi, M. Suzuki and T.T. Yanagida, XENON1T anomaly and its implication for decaying warm dark matter, Phys. Lett. B 811 (2020) 135976 [arXiv:2006.12348] [INSPIRE].

[16] G. Paz, A.A. Petrov, M. Tammaro and J. Zupan, Shining dark matter in Xenon1T, arXiv:2006.12462 [INSPIRE].

[17] A. Bally, S. Jana and A. Trautner, Neutrino self-interactions and XENON1T electron recoil excess, Phys. Rev. Lett. 125 (2020) 161802 [arXiv:2006.11919] [INSPIRE].

[18] B. Batell, M. Pospelov and A. Ritz, Direct detection of multi-component secluded WIMPs, Phys. Rev. D 79 (2009) 115019 [arXiv:0903.3396] [InSPIRE].

[19] P.W. Graham, R. Harnik, S. Rajendran and P. Saraswat, Exothermic dark matter, Phys. Rev. D 82 (2010) 063512 [arXiv: 1004.0937] [INSPIRE].

[20] R. Essig, J. Kaplan, P. Schuster and N. Toro, On the origin of light dark matter species, arXiv: 1004.0691 [INSPIRE].

[21] J. Smirnov and J.F. Beacom, New freezeout mechanism for strongly interacting dark matter, Phys. Rev. Lett. 125 (2020) 131301 [arXiv: 2002.04038] [INSPIRE].

[22] R. Essig, M. Fernandez-Serra, J. Mardon, A. Soto, T. Volansky and T.-T. Yu, Direct detection of sub-GeV dark matter with semiconductor targets, JHEP 05 (2016) 046 [arXiv: 1509.01598] [INSPIRE].

[23] B.M. Roberts, V.A. Dzuba, V.V. Flambaum, M. Pospelov and Y.V. Stadnik, Dark matter scattering on electrons: accurate calculations of atomic excitations and implications for the DAMA signal, Phys. Rev. D 93 (2016) 115037 [arXiv:1604.04559] [INSPIRE].

[24] R. Essig, J. Mardon and T. Volansky, Direct detection of Sub-GeV dark matter, Phys. Rev. D 85 (2012) 076007 [arXiv: 1108.5383] [INSPIRE].

[25] R. Essig, A. Manalaysay, J. Mardon, P. Sorensen and T. Volansky, First direct detection limits on sub-GeV dark matter from XENON10, Phys. Rev. Lett. 109 (2012) 021301 [arXiv:1206.2644] [INSPIRE].

[26] R. Essig, T. Volansky and T.-T. Yu, New constraints and prospects for sub-GeV dark matter scattering off electrons in Xenon, Phys. Rev. D 96 (2017) 043017 [arXiv: 1703.00910] [INSPIRE].

[27] G. Jungman, M. Kamionkowski and K. Griest, Supersymmetric dark matter, Phys. Rept. 267 (1996) 195 [hep-ph/9506380] [INSPIRE].

[28] A. Carrillo-Monteverde, Y.-J. Kang, H.M. Lee, M. Park and V. Sanz, Dark matter direct detection from new interactions in models with spin-two mediators, JHEP 06 (2018) 037 [arXiv: 1803.02144] [INSPIRE].

[29] K. Griest and D. Seckel, Three exceptions in the calculation of relic abundances, Phys. Rev. D 43 (1991) 3191 [INSPIRE].

[30] R.T. D'Agnolo and J.T. Ruderman, Light dark matter from forbidden channels, Phys. Rev. Lett. 115 (2015) 061301 [arXiv:1505.07107] [INSPIRE]. 
[31] S.-M. Choi, Y.-J. Kang and H.M. Lee, On thermal production of self-interacting dark matter, JHEP 12 (2016) 099 [arXiv: 1610.04748] [INSPIRE].

[32] Y.-J. Kang and H.M. Lee, Lightening gravity-mediated dark matter, Eur. Phys. J. C 80 (2020) 602 [arXiv: 2001.04868] [INSPIRE].

[33] S.-M. Choi, J. Kim, H.M. Lee and B. Zhu, Connecting between inflation and dark matter in models with gauged $Z_{3}$ symmetry, JHEP 06 (2020) 135 [arXiv:2003.11823] [INSPIRE].

[34] H.M. Lee, C.B. Park and M. Park, Supersymmetric Higgs-portal and X-ray lines, Phys. Lett. B $\mathbf{7 4 4}$ (2015) 218 [arXiv:1501.05479] [INSPIRE].

[35] C.B. Jackson, G. Servant, G. Shaughnessy, T.M.P. Tait and M. Taoso, Gamma-ray lines and one-loop continuum from s-channel dark matter annihilations, JCAP 07 (2013) 021 [arXiv:1302.1802] [INSPIRE].

[36] C.B. Jackson, G. Servant, G. Shaughnessy, T.M.P. Tait and M. Taoso, Gamma rays from top-mediated dark matter annihilations, JCAP 07 (2013) 006 [arXiv: 1303.4717] [INSPIRE].

[37] M. Drewes et al., A white paper on keV sterile neutrino dark matter, JCAP 01 (2017) 025 [arXiv: 1602.04816] [INSPIRE].

[38] SuPER-KamiokAnde collaboration, Search for dark matter WIMPs using upward through-going muons in Super-Kamiokande, Phys. Rev. D 70 (2004) 083523 [Erratum ibid. 70 (2004) 109901] [hep-ex/0404025] [INSPIRE].

[39] L. Covi, M. Grefe, A. Ibarra and D. Tran, Neutrino signals from dark matter decay, JCAP 04 (2010) 017 [arXiv:0912.3521] [INSPIRE].

[40] Super-Kamiokande collaboration, Supernova relic neutrino search with neutron tagging at Super-Kamiokande-IV, Astropart. Phys. 60 (2015) 41 [arXiv:1311.3738] [InSPIRE].

[41] Super-Kamiokande collaboration, Measurements of the atmospheric neutrino flux by Super-Kamiokande: energy spectra, geomagnetic effects, and solar modulation, Phys. Rev. D 94 (2016) 052001 [arXiv: 1510.08127] [INSPIRE].

[42] P. Bandyopadhyay, E.J. Chun and R. Mandal, Feeble neutrino portal dark matter at neutrino detectors, JCAP 08 (2020) 019 [arXiv:2005.13933] [INSPIRE].

[43] BABAR collaboration, Search for a dark photon in $e^{+} e^{-}$collisions at BaBar, Phys. Rev. Lett. 113 (2014) 201801 [arXiv:1406.2980] [INSPIRE].

[44] BABAR collaboration, Search for invisible decays of a dark photon produced in $e^{+} e^{-}$ collisions at BaBar, Phys. Rev. Lett. 119 (2017) 131804 [arXiv:1702.03327] [INSPIRE].

[45] J.D. Bjorken et al., Search for neutral metastable penetrating particles produced in the SLAC beam dump, Phys. Rev. D 38 (1988) 3375 [INSPIRE].

[46] B. Batell, R. Essig and Z. Surujon, Strong constraints on Sub-GeV dark sectors from SLAC beam dump E137, Phys. Rev. Lett. 113 (2014) 171802 [arXiv:1406.2698] [INSPIRE].

[47] NA64 collaboration, Search for invisible decays of sub-GeV dark photons in missing-energy events at the CERN SPS, Phys. Rev. Lett. 118 (2017) 011802 [arXiv:1610. 02988] [INSPIRE].

[48] D. Banerjee et al., Dark matter search in missing energy events with NA64, Phys. Rev. Lett. 123 (2019) 121801 [arXiv:1906.00176] [INSPIRE]. 
[49] Y. Hochberg, E. Kuflik, T. Volansky and J.G. Wacker, Mechanism for thermal relic dark matter of strongly interacting massive particles, Phys. Rev. Lett. 113 (2014) 171301 [arXiv: 1402.5143] [INSPIRE].

[50] Y. Hochberg, E. Kuflik, H. Murayama, T. Volansky and J.G. Wacker, Model for thermal relic dark matter of strongly interacting massive particles, Phys. Rev. Lett. 115 (2015) 021301 [arXiv: 1411.3727] [INSPIRE].

[51] H.M. Lee and M.-S. Seo, Communication with SIMP dark mesons via $Z^{\prime}$-portal, Phys. Lett. B 748 (2015) 316 [arXiv:1504.00745] [INSPIRE].

[52] S.-M. Choi, H.M. Lee and M.-S. Seo, Cosmic abundances of SIMP dark matter, JHEP 04 (2017) 154 [arXiv:1702.07860] [INSPIRE].

[53] S.-M. Choi et al., Vector SIMP dark matter, JHEP 10 (2017) 162 [arXiv:1707.01434] [INSPIRE].

[54] S.-M. Choi, H.M. Lee, P. Ko and A. Natale, Resolving phenomenological problems with strongly-interacting-massive-particle models with dark vector resonances, Phys. Rev. D 98 (2018) 015034 [arXiv: 1801.07726] [INSPIRE].

[55] S.-M. Choi, H.M. Lee, Y. Mambrini and M. Pierre, Vector SIMP dark matter with approximate custodial symmetry, JHEP 07 (2019) 049 [arXiv: 1904.04109] [INSPIRE].

[56] S.-M. Choi and H.M. Lee, SIMP dark matter with gauged $Z_{3}$ symmetry, JHEP 09 (2015) 063 [arXiv: 1505.00960] [INSPIRE].

[57] H.M. Lee, M. Park and W.-I. Park, Axion-mediated dark matter and Higgs diphoton signal, JHEP 12 (2012) 037 [arXiv:1209.1955] [INSPIRE]. 